PROCEEDINGS OF THE

AMERICAN MATHEMATICAL SOCIETY

Volume 131, Number 10, Pages 3063-3066

S 0002-9939(03)07078-3

Article electronically published on March 11, 2003

\title{
RANGE OF THE GRADIENT OF A SMOOTH BUMP FUNCTION IN FINITE DIMENSIONS
}

\author{
LUDOVIC RIFFORD
}

(Communicated by Jonathan M. Borwein)

\begin{abstract}
This paper proves the semi-closedness of the range of the gradient for sufficiently smooth bumps in the Euclidean space.
\end{abstract}

Let $\mathbb{R}^{N}$ be the Euclidean space of dimension $N$. A bump on $R^{N}$ is a function from $\mathbb{R}^{N}$ into $\mathbb{R}$ with a bounded nonempty support. The aim of this short paper is to partially answer an open question suggested by Borwein, Fabian, Kortezov and Loewen in [1]. Let $f: \mathbb{R}^{N} \rightarrow \mathbb{R}$ be a $C^{1}$-smooth bump function; does $f^{\prime}\left(\mathbb{R}^{N}\right)$ equal the closure of its interior? We are not able to provide an answer, but we can prove the following result.

Theorem 0.1. Let $f: \mathbb{R}^{N} \rightarrow \mathbb{R}$ be a $C^{N+1}$-smooth bump. Then $f^{\prime}\left(\mathbb{R}^{N}\right)$ is the closure of its interior.

We do not know if the hypothesis on the regularity of the bump $f$ is optimal in our theorem when $N \geq 3$. However, the result can be improved for $N=2$; Gaspari [3] proved by specific two-dimensional arguments that the conclusion holds if the bump is only assumed to be $C^{2}$-smooth on the plane. Again we cannot say if we need the bump function to be $C^{2}$ for $N=2$. We proceed now to prove our theorem.

\section{Proof of Theorem 0.1}

For the sequel, we set $F:=f^{\prime}=\nabla f$. Moreover, since the theorem is obvious for $N=1$ we will assume that $N \geq 2$. The proof is based on a refinement of Sard's Theorem that can be found in Federer [2]. Let us denote by $B_{k}, C_{k}(k \in\{0, \cdots, N\})$ the sets defined as follows:

$$
\begin{aligned}
& B_{k}:=\left\{x \in \mathbb{R}^{N}: \operatorname{rank} D F(x) \leq k\right\}, \\
& C_{k}:=\left\{x \in \mathbb{R}^{N}: \operatorname{rank} D F(x)=k\right\} .
\end{aligned}
$$

Of course $C_{k} \subseteq B_{k}$ and $B_{N}=\mathbb{R}^{N}$. Theorem 3.4.3 in 2$]$ says that if the function $F$ is $C^{N}$-smooth, then for all $k=0, \cdots, N-1$,

$$
\mathcal{H}^{k+1}\left(F\left(B_{k}\right)\right)=\mathcal{H}^{k+1}\left(F\left(C_{k}\right)\right)=0,
$$

where $\mathcal{H}^{k+1}$ denotes the $(k+1)$-dimensional Hausdorff measure.

Received by the editors April 16, 2002.

2000 Mathematics Subject Classification. Primary 46G05, 58C25.

Key words and phrases. Smooth bump, gradient. 
Fix $\bar{x}$ in $\mathbb{R}^{N}$ and let us prove that $F(\bar{x})$ belongs to the $\operatorname{closure}$ of $\operatorname{int}\left(F\left(\mathbb{R}^{N}\right)\right)$. Since it is well known that $0 \in \operatorname{int}\left(F\left(\mathbb{R}^{N}\right)\right.$ ) (see Wang [6]), we can assume that $F(\bar{x}) \neq 0$. Our proof begins with the following lemma.

Lemma 1.1. There exists a neighbourhood $\mathcal{V}$ of $F(\bar{x})$ relative to $F\left(\mathbb{R}^{N}\right)$ and an integer $\bar{k} \in\{1, \cdots, N\}$ such that for any $x \in F^{-1}(\mathcal{V}), \operatorname{rank} D F(x) \leq \bar{k}$ and there exists a sequence $\left(v_{n}\right)_{n \in \mathbb{N}}$ in $\mathcal{V}$ which converges to $F(\bar{x})$ such that

$$
F^{-1}\left(v_{n}\right) \subseteq \operatorname{int}\left(C_{\bar{k}}\right) .
$$

Proof. Let us fix $V$ an open neighbourhood of $F(\bar{x})$ relative to $F\left(\mathbb{R}^{N}\right)$ and denote by $k_{0}$ the max of the $k$ 's in $\{0,1, \cdots, n\}$ which satisfy $V \cap F\left(C_{k}\right) \neq \emptyset$.

First of all we remark that $k_{0}>0$. As a matter of fact, suppose that for any $k \geq 1, V \cap F\left(C_{k}\right)=\emptyset$, that is, for any $y$ in $F^{-1}(V)$, rank $D F(y)=0$. Since $F^{-1}(V)$ is open this implies that $F$ is constant on $F^{-1}(V)$ and hence that $F(\bar{x})$ is isolated in $F\left(\mathbb{R}^{N}\right)$. So, we get a contradiction by arc-connectedness of $F\left(\mathbb{R}^{N}\right)$ (and since $F(\bar{x}) \neq 0$ and $0 \in F\left(\mathbb{R}^{n}\right)$ ). Consequently, we deduce that there exists $y \in \mathbb{R}^{N}$ such that $F(y) \in V$ and $\operatorname{rank} D F(y)=k_{0}>0$. Furthermore for all $z \in F^{-1}(V), \operatorname{rank} D F(z) \leq k_{0}$. Hence by lower semicontinuity of $z \mapsto$ $\operatorname{rank} D F(z)$, this implies that $\operatorname{rank} D F$ is constant in a neighbourhood of $y$ (because $\left\{z: \operatorname{rank} D F(z) \geq k_{0}\right\}$ is open). Therefore, by the rank theorem (see Rudin [4, Theorem 9.20]), $V$ has the structure of a $k_{0}$-dimensional manifold near $F(y)$, and hence $\mathcal{H}^{k_{0}}(V)>0$. Thus by (1.1), $V \backslash F\left(B_{k_{0}-1}\right)$ is nonempty. We conclude that for any $v$ in the latter set,

$$
F(z)=v \Longrightarrow \operatorname{rank} D F(z)=k_{0} ;
$$

in addition $z$ has a neighbourhood on which $\operatorname{rank} D F \leq \bar{k}$ by choice of $\bar{k}$, and the set where rank $D F \geq \bar{k}$ is open. Consequently such a $v$ satisfies $F^{-1}(v) \subseteq \operatorname{int}\left(C_{\bar{k}}\right)$. Repeating this argument with a decreasing sequence on neighbourhoods, we get a decreasing sequence of $k_{0}$-values in $\{1, \cdots, n\}$ which has to be stationary. Hence the proof is easy to complete.

We now claim the following lemma.

Lemma 1.2. The constant of Lemma 1.1] satisfies $\bar{k}=N$.

Proof. Let us remark that since $F=f^{\prime}=\nabla f$, the Jacobian of $F$ at any point $y$ in $\mathbb{R}^{N}$ is actually the Hessian of the function $f$. We argue by contradiction and so we assume that $\bar{k}<N$.

By the previous remark, for any $y \in \mathbb{R}^{N}, D F(y)$ is a symmetric matrix, the nontrivial vector subspaces $\operatorname{Ker} D F(y)$ and $\operatorname{Im} D F(y)$ are orthogonal, and $D F(y)$ induces an automorphism on $\operatorname{Im} D F(y)$. Let us fix $n \in \mathbb{N}$. By Lemma 1.1 and by the constant rank theorem (see for instance Spivak 5 page 65 ) we deduce that $M_{n}:=\left\{y: F(y)=v_{n}\right\}$ is a submanifold of $\mathbb{R}^{N}$ of dimension $N-k$ and at least $C^{2}$-smooth (since $F$ is $C^{N}$-smooth and $N \geq 2$ ). Furthermore since $f$ is a bump, $M_{n}$ is a compact submanifold.

Now since $M_{n}$ is a $C^{2}$ submanifold of $\mathbb{R}^{N}$ there exists an open tubular neighbourhood $\mathcal{U} \subset \mathcal{V}$ of $M_{n}$ and a $C^{2}$-smooth function $r: \mathcal{U} \rightarrow M_{n}$ which is the projection on the set $M_{n}$ such that for any $x \in \mathcal{U}, x-r(x) \in N_{r(x)} M_{n}$, where for any $p \in M_{n}, N_{p} M_{n}$ denotes the normal space of $M_{n}$ at $p$. In addition, from the properties of the constant $\bar{k}$, by reducing $\mathcal{U}$ if necessary, we can assume that for 
any $x \in \mathcal{U}$, $\operatorname{rank} D F(x)=\bar{k}$. We set the following function on the neighbourhood $\mathcal{U}:$

$$
\begin{aligned}
\Phi: \mathcal{U} & \rightarrow \mathbb{R}^{N}, \\
x & \mapsto D F(r(x))(x-r(x)) .
\end{aligned}
$$

We now need the following result.

Lemma 1.3. If $M_{n}$ is a compact $C^{2}$ submanifold of $\mathbb{R}^{N}$, then for all $\xi$ in the unit sphere $\mathbb{S}^{N-1}$, there exists $p \in M_{n}$ such that $\xi \in N_{p} M_{n}$.

Proof. Consider for any $l \in \mathbb{N}, p_{l}:=\operatorname{proj}_{M_{n}}(l \xi)$, where $\operatorname{proj}_{M_{n}}(\cdot)$ denotes the projection map on the closed set $M_{n}$. Since the submanifold $M_{n}$ is $C^{2}$, the vector $\frac{l \xi-p_{l}}{\left\|l \xi-P_{l}\right\|}$ belongs to $N_{p_{l}} M_{n}$. Moreover by compactness of $M_{n}$ we can assume that $p_{l} \rightarrow \bar{p}$ when $l$ tends to infinity. Now since the sequence $\left(p_{l}\right)_{l \in \mathbb{N}}$ is bounded, we have that $\lim _{l \rightarrow \infty} \frac{l \xi-p_{l}}{\left\|l \xi-p_{l}\right\|}=\xi$. By continuity of the normal bundle $N M_{n}$, we easily conclude that $\xi \in N_{\bar{p}} M_{n}$.

Returning to the proof of Lemma 1.2, Lemma 1.3 immediately implies that for all $\xi \in \mathbb{S}^{N-1}$, there exists $p \in M_{n}$ and $v \in N_{p} M_{n}$ such that $v=\xi$. Furthermore the map $D F(p)$ is an automorphism on $N_{p} M_{n}$, hence there exists $w \in N_{p} M_{n}$ such that $D F(p)(w)=v$. We conclude that for any $t$ small enough (s.t. $p+t w \in \mathcal{U}$ ), $D F(p)(t w)=t \xi$ and hence that $\Phi(p+t w)=t \xi$. Furthermore since $M_{n}$ is compact and since the map $p \mapsto\left[D F(p)_{\mid N_{p} M_{n}}\right]^{-1}$ is continuous on $M_{n}$, we deduce that $\|w\|$ is bounded above. Hence by compactness on $M_{n}$, we get that for some $t_{0}>0$ the ball $B\left(0, t_{0}\right)$ is included in $\Phi(\mathcal{U})$; hence $\Phi(\mathcal{U})$ has a nonempty interior. Therefore (since the function $\Phi$ is smooth enough) Sard's Theorem gives us the existence of regular values of $\Phi$ in $\mathbb{R}^{N}$. So there exists $\bar{y} \in \mathcal{U}$ such that $\operatorname{rank} D \Phi(\bar{y})=N$. Consequently there exists $\rho>0$ such that the map $\Phi$ is one-to-one on $\mathcal{W}=B(\bar{y}, \rho)$ (the ball centered at $\bar{y}$ with radius $\rho$ ).

For any $l \in \mathbb{N}^{*}$, we set $y_{l}:=r(\bar{y})+\frac{1}{l}(\bar{y}-r(\bar{y}))$. The constant rank theorem implies that for any $l$ the set $V_{l}:=\left\{y \in \mathcal{U}: F(y)=F\left(y_{l}\right)\right\}$ is a submanifold of $\mathcal{U}$ of dimension $N-\bar{k}$. (Of course $V_{l}$ might be noncompact in $\mathcal{U}$, i.e. $\overline{V_{l}}$ not included in $\mathcal{U}$.) On the other hand, by Lipschitz continuity of $D F(\cdot)$ and since $N-\bar{k}>0$, there exists a neighbourhood $\mathcal{Y}$ of the segment $[\bar{y}, r(\bar{y})]$ in $\operatorname{co}\{\mathcal{W} \cup r(\mathcal{W})\}$ and a Lipschitz continuous map $X: \mathcal{Y} \rightarrow \mathbb{R}^{N}$ such that for any $x \in \mathcal{Y}$,

$$
X(x) \in \operatorname{ker} D F(x) \text { and }\|X(x)\|=1 .
$$

If we denote by $\theta_{X}(y, \tau)$ the local flow of the vector field $X$ on $\mathcal{Y}$, we get that for any $\tau$ small enough, $\theta_{X}\left(y_{l}, \tau\right) \in V_{l}$. On the other hand, Gronwall's Lemma easily yields the following (we omit the proof):

Lemma 1.4. There exist two positive constants $K, \mu$ such that for any $l \in \mathbb{N}^{*}$ and for any $\tau \leq \mu$, we have

$$
\begin{aligned}
& \theta_{X}\left(y_{l}, \tau\right) \in \operatorname{co}\left\{B\left(\bar{y}, \frac{\rho}{2}\right) \cup r\left(B\left(\bar{y}, \frac{\rho}{2}\right)\right)\right\} \\
& \frac{\left\|\theta_{X}\left(y_{l}, \tau\right)-r\left(\theta_{X}\left(y_{l}, \tau\right)\right)\right\|}{\left\|y_{l}-r\left(y_{l}\right)\right\|} \in\left[e^{-K \tau}, e^{K \tau}\right] .
\end{aligned}
$$


We now conclude the proof of Lemma 1.2. We set for any $l \in \mathbb{N} \backslash\{0\}, z_{l}:=$ $\theta_{X}\left(y_{l}, \mu\right)$. First remark that if $\mu$ is small enough, then we have (recall that $\|X\|=1$ )

$$
\begin{aligned}
& \left\langle X\left(\theta_{X}\left(y_{l}, s\right)\right), X\left(y_{l}\right)\right\rangle \geq \frac{1}{2} \\
\Longrightarrow & \left\langle\int_{0}^{\mu} X\left(\theta_{X}\left(y_{l}, s\right)\right) d s, X\left(y_{l}\right)\right\rangle \geq \frac{\mu}{2} \\
\Longrightarrow & \left\|z_{l}-y_{l}\right\| \geq \frac{\mu}{2} .
\end{aligned}
$$

By considering a converging subsequence of $\left(z_{l}\right)_{l \in \mathbb{N}^{*}}$ if necessary we can compute

$$
\begin{aligned}
\lim _{l \rightarrow \infty} \frac{F\left(y_{l}\right)-F\left(r\left(y_{l}\right)\right)}{\left\|z_{l}-r\left(z_{l}\right)\right\|} & =\lim _{l \rightarrow \infty} \frac{F\left(z_{l}\right)-F\left(r\left(z_{l}\right)\right)}{\left\|z_{l}-r\left(z_{l}\right)\right\|} \\
& =\lim _{l \rightarrow \infty} D F\left(r\left(z_{l}\right)\right)\left(\frac{z_{l}-r\left(z_{l}\right)}{\left\|z_{l}-r\left(z_{l}\right)\right\|}\right) \\
& =D F(\bar{z})(\bar{\zeta}),
\end{aligned}
$$

where $\lim _{l \rightarrow \infty} z_{l}=\bar{z}=r(\bar{z}) \in M_{n}$ and $\lim _{l \rightarrow \infty} \frac{z_{l}-r\left(z_{l}\right)}{\left\|z_{l}-r\left(z_{l}\right)\right\|}=\bar{\zeta} \in N_{\bar{z}} M_{n}$. We deduce that

$$
\begin{aligned}
D F(r(\bar{y}))(\bar{y}-r(\bar{y})) & =\lim _{l \rightarrow \infty} l\left(F\left(y_{l}\right)-F\left(r\left(y_{l}\right)\right)\right) \\
& =\lim _{l \rightarrow \infty} l\left\|z_{l}-r\left(z_{l}\right)\right\| \frac{F\left(y_{l}\right)-F\left(r\left(y_{l}\right)\right)}{\left\|z_{l}-r\left(z_{l}\right)\right\|} \\
& =c\|\bar{y}-r(\bar{y})\| D F(\bar{z})(\bar{\zeta}) \\
& =D F(\bar{z})(c\|\bar{y}-r(\bar{y})\| \bar{\zeta}),
\end{aligned}
$$

with $c=\lim _{l \rightarrow \infty} \frac{\left\|z_{l}-r\left(z_{l}\right)\right\|}{\left\|y_{l}-r\left(y_{l}\right)\right\|}$.

The computations prove that $\Phi(\bar{y})=\Phi(\bar{z}+c\|\bar{y}-r(\bar{y})\| \bar{\zeta})$. Furthermore by (1.3) and (1.5), $\bar{z}$ belongs to $r(\mathcal{W})$ and $\|\bar{z}-r(\bar{y})\|>0$. Consequently since $\Phi$ is injective on $\mathcal{W}$, it remains to prove that $\bar{z}+c\|\bar{y}-r(\bar{y})\| \bar{\zeta}$ is in $\mathcal{W}$ to get a contradiction. By (1.4) taking $\mu$ smaller if necessary, we get the result of Lemma 1.2

The proof of Theorem 0.1 is now easy. Since $\bar{k}=N$, for any $n \in \mathbb{N}$ the different values $v_{n}$ of Lemma 1.1]belong to the interior of $f^{\prime}\left(\mathbb{R}^{N}\right)$ and moreover the sequence $\left(v_{n}\right)_{n \in \mathbb{N}}$ converges to $F(\bar{x})$. This proves the theorem.

\section{REFERENCES}

1. J. M. Borwein, M. Fabian, I. Kortezov, and P. D. Loewen. The range of the gradient of a continuously differentiable bump. J. Nonlinear Convex Anal., 2(1):1-19, 2001. MR 2002c:58012

2. H. Federer. Geometric measure theory. Springer-Verlag, New York Inc., New York, 1969. MR 41:1976

3. T. Gaspari. On the range of the derivative of a real valued function with bounded support. Preprint.

4. W. Rudin. Principles of mathematical analysis. McGraw-Hill Book Co., New York, 1964. MR 29:3587

5. M. Spivak. A comprehensive introduction to differential geometry. Vol. I. Publish or Perish Inc., Wilmington, Del., second edition, 1979. MR 82g:53003a

6. X. Wang. Pathological examples of Lipschitz functions. Ph.D. thesis, SFU, 1995.

Institut Girard Desargues, Université Claude Bernard Lyon I, 69622 Villeurbanne, FrANCE

E-mail address: rifford@igd.univ-lyon1.fr 